\title{
DYNAMICS OF DRIVEN OSCILLATORS WITH COMPLEX VARIABLES ON LYAPUNOV DIAGRAMS
}

\author{
Holokx A. Albuquerque*, Julio C. D. Cardoso* \\ * Department of Physics \\ Santa Catarina State University \\ Joinville, SC, Brazil
}

Emails: holokx.albuquerque@udesc.br, jcdamore@gmail.com

\begin{abstract}
In this work it is shown the dynamical behavior of driven complex variable oscillators on Lyapunov diagrams, through the computation of the Lyapunov exponents spectrum. Here, we consider the largest and the second largest exponents on Lyapunov diagrams to identify regions of chaotic, quasi-periodic and periodic behaviors. In these diagrams we show the coexistence of limit cycles, chaotic attractors and 2-tori. Periodic and quasi-periodic structures embedded in chaotic regions are also observed on the Lyapunov diagrams.
\end{abstract}

Keywords - Chaotic Dynamics, Driven Oscillators, Complex Variables, Periodic Structures.

Resumo - Neste trabalho reportamos a dinâmica de osciladores forçados de variáveis complexas nos diagramas de Lyapunov, através do cálculo numérico do espectro dos expoentes de Lyapunov. Aqui consideramos o primeiro e o segundo maior expoente nos diagramas de Lyapunov para identificar regions de caos, de quase-periodicidade e de periodicidade. Nestes diagramas mostramos a coexistência de ciclos limites, atratores caóticos e Tori-2. Estruturas periódicas e quase-periódicas imersas em regiões caóticas são também observadas nos diagramas de Lyapunov.

Palavras-chave — Dinâmica Caótica, Osciladores Forçados, Variáveis Complexas, Estruturas Periódicas.

\section{Introduction}

The sensitive dependence on initial conditions is a signature of chaotic behavior (Sprott, 2003). Nowadays in the literature, a well accepted invariant measure that identifies the chaotic motion in nonlinear systems is the Lyapunov exponents (LEs) (Wolf et al., 1985; Eckmann et al., 1986; Marshall and Sprott, 2009). More recently, a growing interest is in the analysis of the LEs on Lyapunov diagrams, where we associate colors for the largest and the second largest exponent varying simultaneously two system's parameters (Rech, 2011; Bonatto et al., 2011; Gallas, 2010; Albuquerque et al., 2008; Celestino et al., 2011; Oliveira and Leonel, 2013; Medeiros et al., 2013; Freire et al., 2012; Zou et al., 2010; Stoop et al., 2012; Stegemann et al., 2011). Motivated by recent works of Marshall and Sprott (Marshall and Sprott, 2009; Marshall and Sprott, 2010), where were proposed simple oscillators with complex variable $z$ that present chaotic behavior, we carry out, in this work, numerical studies of three most representative complex oscillators proposed by Marshall and Sprott (2009), with the introduction of another parameter, $A$. Below, we show the three equations in the $z$ complex variable, the systems (1), (2), and (3).

$$
\begin{gathered}
\dot{z}+z^{2}-\bar{z}+1=A e^{i \Omega t}, \\
\dot{z}+(z-\bar{z}) z+1=A e^{i \Omega t}, \\
\dot{z}+\left(z^{2}-\bar{z}^{2}\right) z+\bar{z}=A e^{i \Omega t},
\end{gathered}
$$

where $\bar{z}$ is the complex conjugate of $z$. Using the explicit form $z=x+i y$, for the real variables $x$ and $y$, we rewrite the non-autonomous systems (1), (2), and (3) as the following autonomous three-dimensional systems,

$$
\begin{gathered}
\dot{x}=-x^{2}+y^{2}+x-1+A \cos (w), \\
\dot{y}=-2 x y-y+A \sin (w), \\
\dot{w}=\Omega . \\
\dot{x}=2 y-1+A \cos (w), \\
\dot{y}=-2 x y+A \sin (w), \\
\dot{w}=\Omega . \\
\dot{x}=4 x y^{2}-x+A \cos (w), \\
\dot{y}=-4 x^{2} y+y+A \sin (w), \\
\dot{w}=\Omega .
\end{gathered}
$$

The main goal here is to construct the Lyapunov diagrams for the systems (4), (5), and (6), varying the parameter pairs $(A, \Omega)$ in a grid of $500 \times 500$ values of parameters. For each pair we calculate the LEs using the algorithm proposed by Wolf et al. (1985) solving the equations by the four-order Runge-Kutta method with time step $1.0 \times 10^{-3}$ and $5.0 \times 10^{6}$ iterations to estimate the LEs for each one of the $2.5 \times 10^{5}$ points in the Lyapunov diagrams. The initial conditions were always initiated at $(0,-0.5,0)$, except for the system (6). As observed by Marshall and Sprott (2009), these systems present multistability regarding with the initialization of the 
systems, therefore, we choose initiate the systems always with the same above initial conditions, for each pair of parameters. The Lyapunov diagrams are constructed with the parameters in each axis and LEs values codified in a colors range. In Section 2 we show the Lyapunov diagrams of the systems (4), (5), and (6), with bifurcation diagrams and attractors, and discussions concerning the results. Section 3 is brief summary.

\section{The Lyapunov Diagrams}

In this section we initiate with the analysis for the system (4). Figure 1 shows the Lyapunov diagram for the largest exponent of the LEs spectrum. Black regions identify periodic behavior, yellow and red ones chaotic behavior. A sequence of periodic structure is observed, where the hookshaped periodic structures organize themselves in a period-adding cascade, as they accumulate in the upper period-1 boundary of the diagram. The period-adding was also observed by Marshall and Sprott (2009), but just analyzing attractors. This period-adding cascade and the upper periodic boundary can be better visualized in the bifurcation diagram of Fig. 2, where it was constructed along the red line of Fig. 1. Figure 3 shows some representative attractors of the periodic structures and chaotic regions. Other feature of the upper period-1 boundary, as can be observed in right side of the bifurcation diagram of Fig. 2, is the bifurcation route to chaos by crisis, from decreasing values of $\Omega$ starting at 1.1 . Similar hook-shaped periodic structures in chaotic regions were also observed by Viana et al. (2010) and Viana et al. (2012), for a forced Chua's circuit.

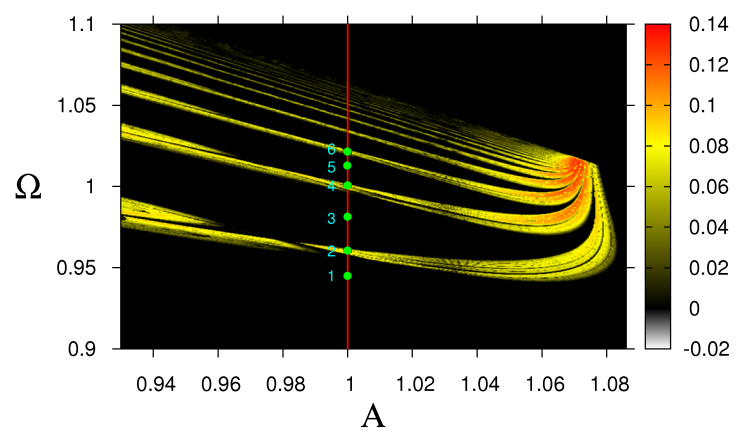

Figure 1: Lyapunov diagram of system (4) for the largest exponent. The right color bar identifies in colors the exponent values. The red line at $A=1$ limits the studies reported by Marshall and Sprott (2009), and identifies the direction of the bifurcation diagram show in Fig. 2. The points market with $1,2,3,4,5$, and 6 represent the pair $(A, \Omega)$ of the attractors in Fig. 3 .

The dynamics of the system (4) on the Lyapunov diagram of Fig. 1 revealed only the presence

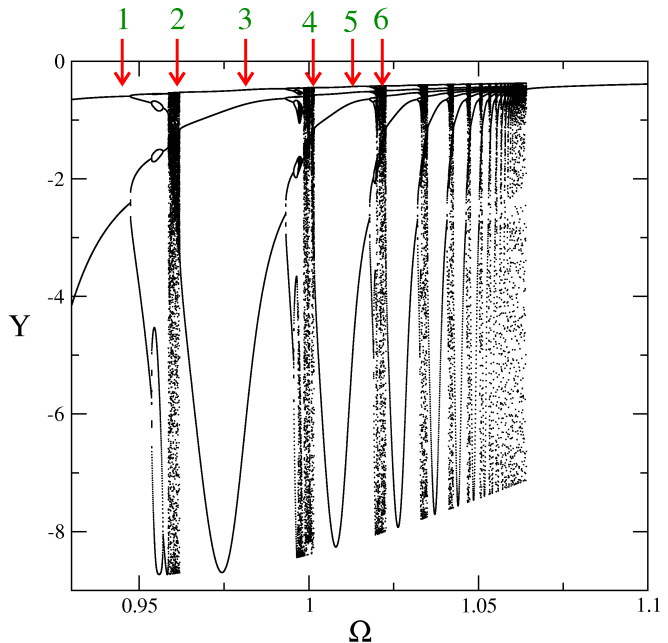

Figure 2: Bifurcation diagram along the red line of Fig. 1, with $0.93 \leq \Omega \leq 1.1$ and $A=1$. The arrows and the numbers indicate the positions of the attractors shown in Fig. 3. The upper periodic boundary is the period- 1 region at the right side of the diagram.

of periodic and chaotic behaviors, at least in the parameters range studied. However, a more rich dynamical behavior is shown by the system (5), where coexist in the same Lyapunov diagram, quasi-periodic, periodic and chaotic behaviors, as already observed by Marshall and Sprott (2009). Figure 4 shows the Lyapunov diagram of the system (5) for the largest exponent of the LEs spectrum, and in Fig. 5 we show the Lyapunov diagram for the second largest exponent. The main reason to present these two Lyapunov diagrams for the two largest exponents of the LEs spectrum of the system (5) is to identify the regions of quasi-periodicity, periodicity and chaos. It is well known that for a three-dimensional system, the LEs spectrum has three values, and for each behavior of the system we can associate it with the signs of the three exponents. For example, for fixed points, the three exponents have negative values, for periodic behavior (limit cycles) one exponent has a null value and the last two have negative values. For quasi-periodic behavior (torus-2) two exponents have null values and the last one has negative value. Finally, for chaotic behavior one exponent has a positive value, one has a null value, and the last one has a negative value. Therefore, for the system (5) if the largest exponent of the LEs spectrum has null or positive value, in the Lyapunov diagram for this largest exponent (Fig. 4) will have black or yellow/red regions, respectively, and the system behavior will be quasi-periodic/periodic or chaotic, respectively. If the second largest exponent of the LEs spectrum for the system (5) has null or negative value, in the Lyapunov diagram for this sec- 


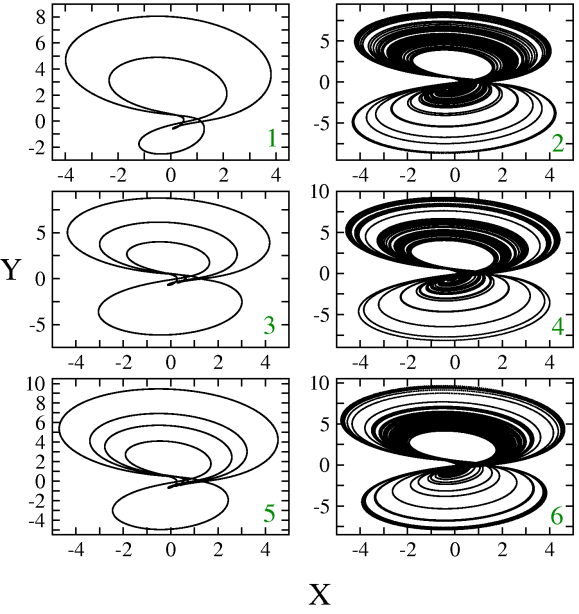

Figure 3: Attractors for the parameters $(A, \Omega)$ indexed by the respective numbers at the bottom right, and that are shown in Figs. 1 and 2.

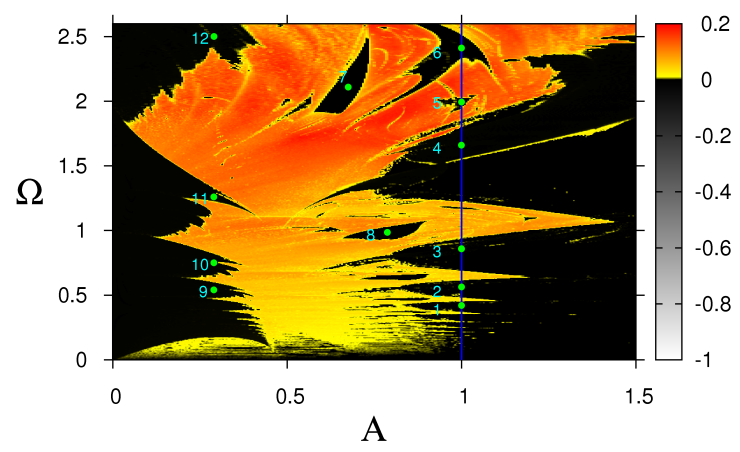

Figure 4: Lyapunov diagram of system (5) for the largest exponent. The right color bar identifies in colors the exponent values. The blue line at $A=1$ limits the studies reported by Marshall and Sprott (2009). The points market with 1 up to 12 , represent the pair $(A, \Omega)$ of the attractor in Fig. 6. Here, black regions are quasi-periodic or periodic behaviors, and yellow/red regions are chaotic behaviors.

ond largest exponent (Fig. 5) will have black or white regions, respectively, and the system behavior will be quasi-periodic/chaotic or periodic, respectively. Summarizing, for the system (5) quasiperiodic behavior is identified by black regions in Fig. 4 and black regions in Fig. 5. Periodic behavior is identified by black regions in Fig. 4 and white regions in Fig. 5. Chaotic behavior is identified by yellow/red regions in Fig. 4 and black regions in Fig. 5. To corroborate, in Fig. 6 we present some attractors for the pair $(A, \Omega)$ located at the points indexed by the numbers in Figs. 4 and 5 .

With Figs. 4, 5, and 6 is possible to localize regions and structures of quasi-periodicity and periodicity embedded in chaotic regions. For example, the attractors 3,4 , and 5 are quasi-periodic attractors (or torus-2 attractors) and they coexist in regions with periodic attractor (limit cycles at-

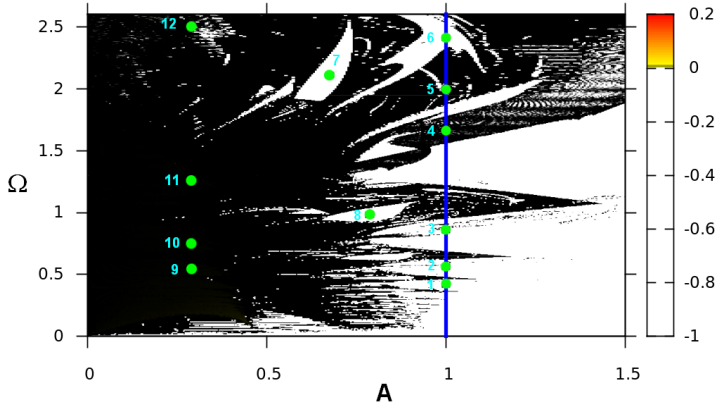

Figure 5: Lyapunov diagram of system (5) for the second largest exponent. The right color bar identifies in colors the exponent values. The blue line at $A=1$ limits the studies reported by Marshall and Sprott (2009). The points market with 1 up to 12 , represent the pair $(A, \Omega)$ of the attractor in Fig. 6. Here, black regions are quasi-periodic or chaotic behaviors, and white regions are periodic behaviors.
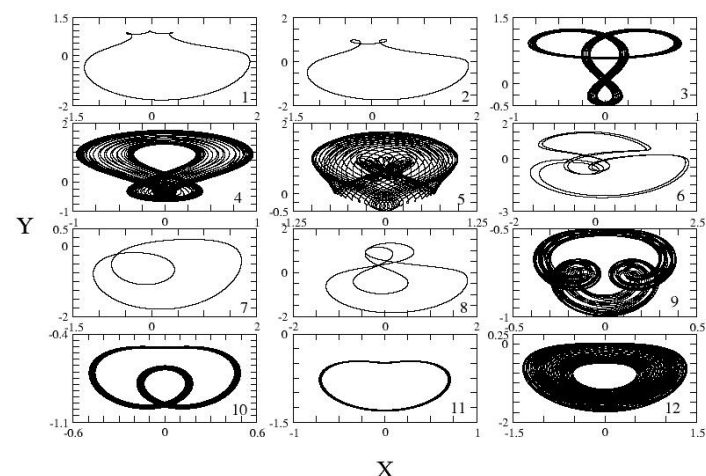

Figure 6: Attractors for the parameters $(A, \Omega)$ indexed by the respective numbers at the bottom right, and that are shown in Figs. 4 and 5.

tractors). An interesting observation is regarding with the quasi-periodic attractor 5. It is localized in a quasi-periodic structure embedded in a chaotic region (see the region with the point 5 in Figs. 4 and 5) once that this structure appears black in Figs. 4 and 5. The attractors 1, 2, 6, 7, and 8 in Fig. 6 are periodic and they are localized in regions and structures of periodicity. The attractors $9,10,11$, and 12 are quasi-periodic attractors and they are localized in regions of quasiperiodicity (see the left side of Figs. 4 and 5). In Table 1 we show some representative values of the LEs spectrum of the attractor of Fig. 6 with the goal to show the good accuracy that we have for compute the LEs spectrum and distinguish the quasi-periodic, periodic and chaotic behaviors on Lyapunov diagrams.

The last system analyzed was the system (6), for which we use the initial conditions $(0,-0.7,0)$. Fig. 7 shows the Lyapunov diagrams for the largest exponent, where black regions are quasi- 
Table 1: Lyapunov exponents for some attractors $(n)$ of Figs. 6, 9 and 12. All Tori listed bellow are related to the quasi-periodic motion. TA, T2, Pe, and Ch mean: type of attractors, torus-2, periodic, and chaotic, respectivelly.

\begin{tabular}{cccccc}
\hline \hline Fig. & $n$ & $\lambda_{1}$ & $\lambda_{2}$ & $\lambda_{3}$ & $\mathrm{TA}$ \\
\hline 6 & 3 & 0 & -0.0000 & -0.0002 & $\mathrm{~T}-2$ \\
6 & 5 & 0.0000 & 0 & -0.0001 & $\mathrm{~T}-2$ \\
6 & 6 & 0 & -0.03 & -0.46 & $\mathrm{Pe}$ \\
6 & 12 & 0.0000 & 0 & -0.0002 & $\mathrm{~T}-2$ \\
9 & 1 & 0 & -0.1532 & -0.1538 & $\mathrm{Pe}$ \\
9 & 2 & 0.086 & 0 & -0.155 & $\mathrm{Ch}$ \\
9 & 3 & 0.0005 & 0 & -0.0010 & $\mathrm{~T}-2$ \\
12 & 5 & 0 & -0.060 & -0.096 & $\mathrm{Pe}$ \\
12 & 6 & 0.19 & 0 & -0.27 & $\mathrm{Ch}$ \\
\hline \hline
\end{tabular}

periodic or periodic behaviors, and yellow/red regions are chaotic behaviors. As previously commented, black regions in this diagram can be periodic or quasi-periodic behaviors, yellow/red regions are chaotic behavior. In Fig. 8 we present

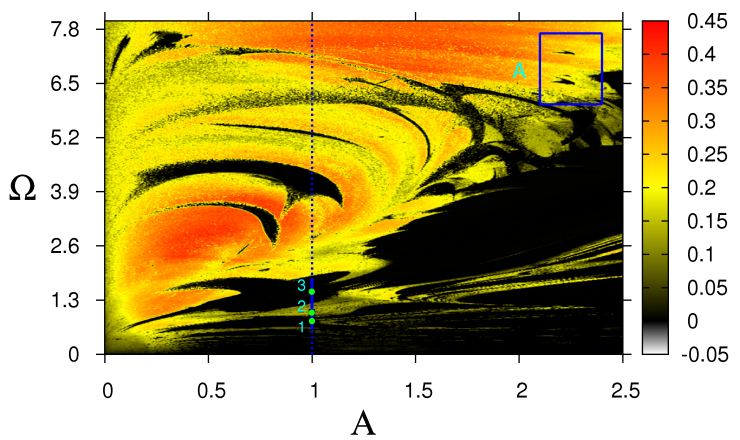

Figure 7: Lyapunov diagram of system (6) for the largest exponent. The right color bar identifies in colors the exponent values. The dotted blue line at $A=1$ limits the studies reported by Marshall and Sprott (2009). The solid blue line, also at $A=1$, represents the direction of the bifurcation diagram of Fig. 8. The points market with 1 , 2 , and 3 , represent the pair $(A, \Omega)$ of the attractor in Fig. 9. Here, black regions are quasi-periodic or periodic behaviors, and yellow/red regions are chaotic behaviors.

the bifurcation diagram along the solid blue line at $A=1$ in Fig. 7, and in Fig. 9 we show three attractors in different positions along the blue line in Fig. 7. Three regions with distinct behaviors can be observed in the bifurcation diagram and corroborated by the attractors of Fig. 9, namely periodic behavior (attractor 1 ), chaotic behavior (attractor 2), and quasi-periodic behavior (attractor 3 ). We observe that the quasi-periodic and periodic behaviors are indistinguishable on Lyapunov diagram for the largest exponent, because both have a null largest exponent (see the points 1 and 3 in Fig. 7). However, in the bifurcation diagram, Fig. 8, the differences comes clear, once that the

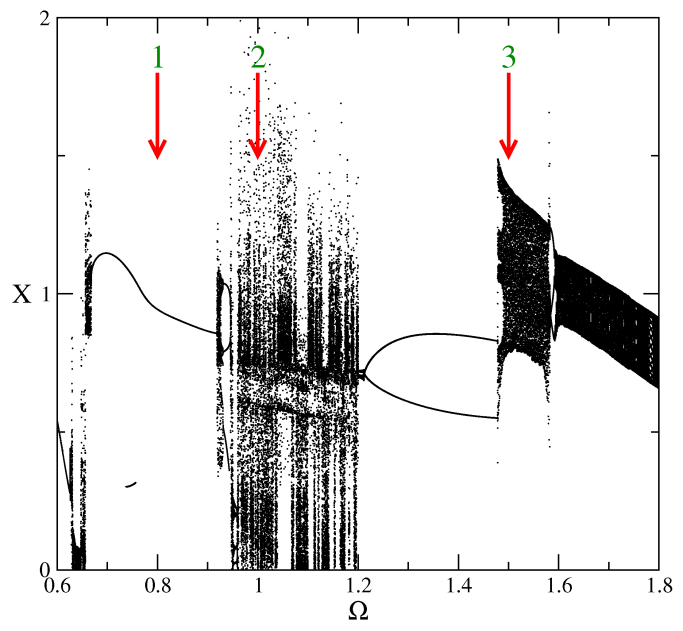

Figure 8: Bifurcation diagram along the solid blue line of Fig. 7 , with $0.6 \leq \Omega \leq 1.8$ and $A=1$. The arrows and the numbers indicate the positions of the attractors shown in Fig. 9.

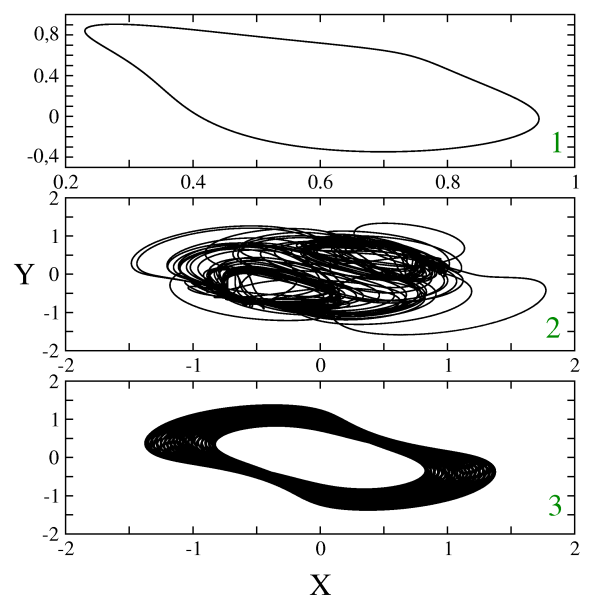

Figure 9: Attractors for the parameters $(A, \Omega)$ indexed by the respective numbers at the bottom right, and that are shown in Figs. 7 and 8 .

periodic behaviors appear as a set of points forming sharp thin lines (the region around the index 1), and quasi-periodic behaviors appear as a set of compact and dense points but with sharp borders (the region around the index 3 ) instead of chaotic behaviors, where the set of points is dense but not compact with unlimited borders (the region around the index 2). The quantitative differences appear in the LEs spectrum of these three attractors, as seen in Table 1. An interesting pattern of periodic structures can be seen in the region inside the box A of Fig. 7. The zooming of this box is shown in Fig. 10, where a set of periodic structures with the same shape are seen. Fig. 11 shows a bifurcation diagram along the blue line of Fig. 10 that cross the two main periodic structures and a third one between the two. Fig. 12 shows the attractor indexed by the numbers 4 up 


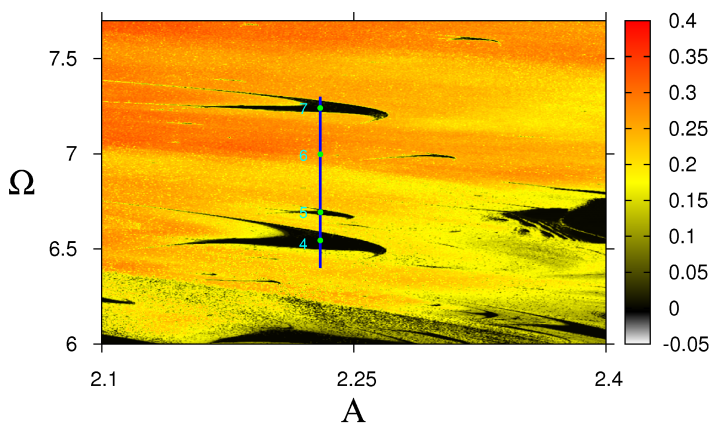

Figure 10: Lyapunov diagram of the box A of Fig. 7 for the largest exponent. The right color bar identifies in colors the exponent values. The blue line at $A=2.24$ represents the direction of the bifurcation diagram of Fig. 11. The points market with 4 up to 7 , represent the pair $(A, \Omega)$ of the attractor in Fig. 12. Here, black regions are periodic behaviors, and yellow/red regions are chaotic behaviors.

to 7 along the blue line of Fig. 10, and their positions by red arrows on the bifurcation diagram of Fig. 11. Table 1 shows the LEs spectrum for the attractors 5 and 6 . These attractors have a very complex topology, and in the bifurcation diagram, we can observe the high-periodicity of the attractors 4,5 , and 7 . Observing the attractors 4 and 5 , and their positions on the bifurcation diagram, it seems that a period-adding occurs from attractor 4 to attractor 5 .

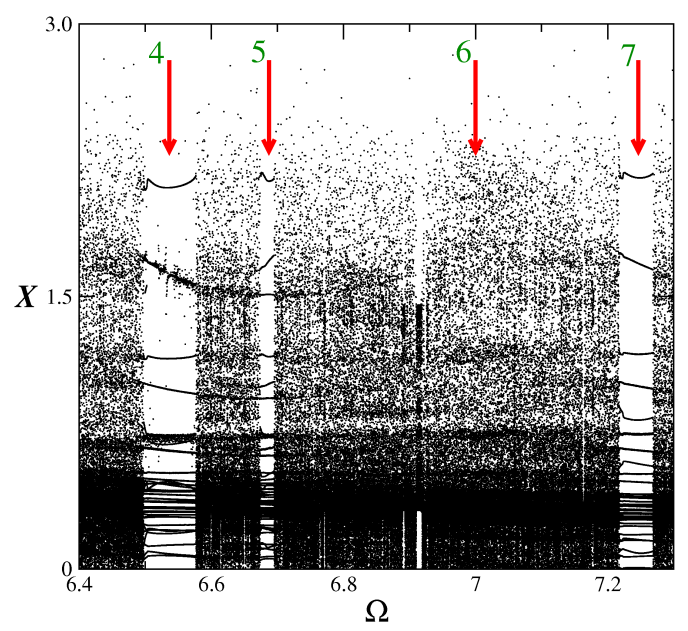

Figure 11: Bifurcation diagram along the blue line of Fig. 10, with $6.4 \leq \Omega \leq 7.3$ and $A=2.24$. The arrows and the numbers indicate the positions of the attractors shown in Fig. 12.

\section{Summary}

By the use of the LEs spectrum, we constructed Lyapunov diagrams with the largest and the second largest exponent to show the rich dynamics of

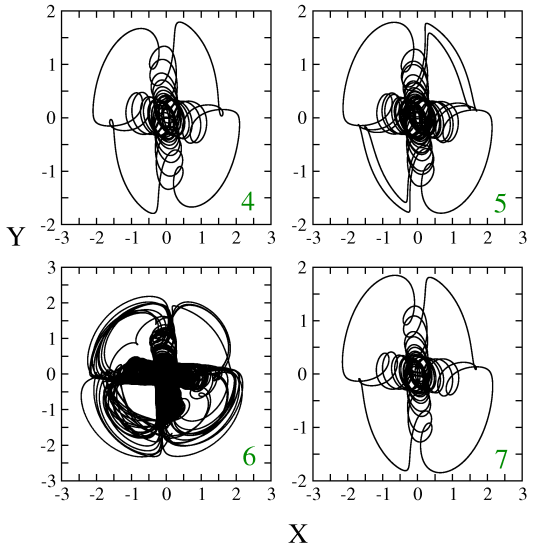

Figure 12: Attractors for the parameters $(A, \Omega)$ indexed by the respective numbers at the bottom right, and that are shown in Figs. 10 and 11.

three driven oscillator models which the three differential equations with real variables can be described by a single differential equation with complex variable. The previously reported oscillators are one-parameter models, and in our work we introduced a new parameter, such that now the oscillators are two-parameter models. With this procedure was possible to study their dynamical behavior on Lyapunov diagrams.

Varying simultaneously the two parameters $(A, \Omega)$ of the systems $(1),(2)$, and (3), was possible to observe on the Lyapunov diagrams regions of quasi-periodicity and periodicity immersed in chaotic regions. The bifurcation diagrams were also used as well as the attractors to corroborate the assumptions provided by the Lyapunov diagrams.

The method of compute the LEs spectrum and study the dynamics of systems in the Lyapunov diagrams constructed with the largest and with the second largest exponent proved to be powerful to characterize the dynamics of nonlinear systems.

\section{Acknowledgment}

The authors thank Conselho Nacional de Desenvolvimento Científico e Tecnológico-CNPq, Coordenação de Aperfeiçoamento de Pessoal de Nível Superior-CAPES, Fundação de Amparo à Pesquisa e Inovação do Estado de Santa CatarinaFAPESC, Brazilian agencies, for financial support, and P. C. Rech for his help in the bifurcation diagram routine.

\section{References}

Albuquerque, H. A., Rubinger, R. M. and Rech, P. C. (2008). Self-similar structures in a 2D 
parameter-space of an inductorless Chua's circuit, Physics Letters A 372: 4793-4798. DOI: $10.1016 /$ j.physleta.2008.05.036

Bonatto, C., Garreau, J. C. and Gallas, J. A. C. (2011). Self-similarities in the frequencyamplitude space of a loss-modulated $\mathrm{CO}_{2}$ laser, Physical Review Letters 375: 1461.

Celestino, A., Manchein, C., Albuquerque, H. and Beims, M. (2011). Ratchet transport and periodic structures in parameter space, Physical Review Letters 106: 234101. DOI: 10.1103/PhysRevLett.106.234101

Eckmann, J.-P., Kamphorst, S. O. and Ciliberto, S. (1986). Liapunov exponents from time se-ries, Physical Review A 34: 4971. DOI: 10.1103/PhysRevA.34.4971

Freire, J. G., Pöschel, T. and Gallas, J. A. C. (2012). Stern-Brocot trees in spiking and bursting of sigmoidal maps, Europhysics Letters 100: 48002 . 10.1209/0295-5075/100/48002

Gallas, J. A. C. (2010). The structure of infinite periodic and chaotic hub cascades in phase diagrams of simple autonomous flows, International Journal of Bifurcation and Chaos 20: 197. DOI: 10.1142/S0218127410025636

Marshall, D. and Sprott, J. C. (2009). Simple driven cahotic oscillators with complex vari-ables, Chaos 19: 013124. DOI: $10.1063 / 1.3080193$

Marshall, D. and Sprott, J. C. (2010). Simple conservative, autonomous, second-order chaotic complex variables systems, International Journal of Bifurcation and Chaos 20: 697. DOI: 10.1142/S0218127410025983

Medeiros, E. S., Medrano-T, R. O., Caldas, I. L. and Souza, S. L. T. D. (2013). Torsionadding and asymptotic winding number for periodic window sequences, Physics Letters A 377: 628-631. DOI: 10.1016/j.physleta.2013.01.004

Oliveira, D. F. M. and Leonel, E. D. (2013). Some dynamical properties of a classical dissipative bouncing ball model with two nonlinearities, Physica A 392: 1762-1769. DOI: 10.1016/j.physa.2012.12.021

Rech, P. C. (2011). Dynamics of a neuron model in different two-dimensional parameter-spaces, Physics Letters A 375: 1461. DOI: 10.1016/j.physleta.2011.02.037

Sprott, J. C. (2003). Chaos and Time-Series Analysis, Oxford University Press.

Stegemann, C., Albuquerque, H. A., Rubinger, R. M. and Rech, P. C. (2011). Lyapunov exponent diagrams of a 4-dimensional Chua system, Chaos 21: 033105. DOI: 10.1063/1.3615232

Stoop, R., Martignoli, S., Benner, P., Stoop, R. and Uwate, Y. (2012). Shrimps: occurrence, scaling and relevance, International Journal of Bifurcation and Chaos 22: 1230032. DOI: 10.1142/S0218127412300327
Viana, E. R., Rubinger, R. M., Albuquerque, H. A., de Oliveira, A. G. and Ribeiro, G. M. (2010). High-resolution parameter space of an experimental chaotic circuit, Chaos 20: 023110. DOI: 10.1063/1.3407482

Viana, E. R., Rubinger, R. M., Albuquerque, H. A., Dias, F. O., de Oliveira, A. G. and Ribeiro, G. M. (2012). Periodicity detection on the parameter-space of a forced Chua's circuit, Nonlinear Dynamics 67: 385. DOI: 10.1007/s11071-011-9986-5

Wolf, A., Swift, J. B., Swinney, H. L. and Vastano, J. A. (1985). Determining Lyapunov exponents from a time series, Physica D 16: 285. DOI: 10.1016/0167-2789(85)90011-9

Zou, Y., Donner, R. V., Donges, J. F., Marwan, N. and Kurths, J. (2010). Identifying complex periodic windows in continuous-time dynamical systems using recurrence-based methods, Chaos 20: 043130. DOI: 10.1063/1.3523304 